\title{
Kelvin-Helmholtz versus Hall magnetoshear instability in astrophysical flows
}

\author{
Daniel O. Gómez* and Cecilia Bejarano \\ Instituto de Astronomía y Física del Espacio, C.C. 67 Suc. 28, 1428 Buenos Aires, Argentina \\ Pablo D. Mininni \\ Departamento de Física, Facultad de Ciencias Exactas y Naturales, Universidad de Buenos Aires \& Instituto de Física de Buenos Aires, \\ Ciudad Universitaria, 1428 Buenos Aires, Argentina
}

(Received 18 November 2013; revised manuscript received 7 April 2014; published 23 May 2014; publisher error corrected 18 June 2014 )

\begin{abstract}
We study the stability of shear flows in a fully ionized plasma. Kelvin-Helmholtz is a well-known macroscopic and ideal shear-driven instability. In sufficiently low-density plasmas, also the microscopic Hall magnetoshear instability can take place. We performed three-dimensional simulations of the Hall-magnetohydrodynamic equations where these two instabilities are present, and carried out a comparative study. We find that when the shear flow is so intense that its vorticity surpasses the ion-cyclotron frequency of the plasma, the Hall magnetoshear instability is not only non-negligible, but it actually displays growth rates larger than those of the Kelvin-Helmholtz instability.
\end{abstract}

DOI: 10.1103/PhysRevE.89.053105

PACS number(s): 95.30.Qd, 47.27.-i, 52.35.Py

\section{INTRODUCTION}

Even though the large-scale behavior of most astrophysical plasmas is well described using magnetohydrodynamics (MHD), at sufficiently smaller scales nonfluidistic effects might become relevant. For instance, when in a fully ionized hydrogen plasma we reach scales as small as the ion skin depth $c / \omega_{p i}$ ( $c$ being the speed of light and $\omega_{p i}$ the ion plasma frequency), the Hall effect becomes non-negligible. This is often the case in various dynamical processes taking place in low-density plasmas, such as in the interstellar medium. Since astrophysical flows are also characterized by very large Reynolds numbers, this in turn implies that a wide range of spatial scales are relevant to properly describe their dynamical behavior all the way from the macroscopic size of the problem to intermediate scales such as $c / \omega_{p i}$ and down to scales small enough where energy eventually dissipates. The role played by the Hall effect in a variety of astrophysical flows has been studied extensively in the literature. The role of the Hall current in turbulent regimes [1], its relevance in the generation of magnetic fields by dynamo action [2,3], or its importance in magnetic reconnection [4,5], are only a few examples.

At sufficiently small scales, the large-scale dynamics is usually perceived as a macroscopic velocity gradient, and it is often modeled through a shear flow. The existence of shear flows is ubiquitous in astrophysics. It is of interest in a variety of problems such as astrophysical jets propagating in the interstellar medium [6], zonal flows being formed in the atmospheres of rotating planets like Jupiter [7], or in the interaction of solar CMEs with the interplanetary medium [8]. The stability of shear flows has been extensively studied and reviewed in the pioneering work of Chandrasekhar (see Ref. [9]). It has also been studied in a variety of astrophysical problems, such as jet collimation [10], the dynamics of spiral

\footnotetext{
*dgomez@df.uba.ar; Also at: Departamento de Física, Facultad de Ciencias Exactas y Naturales, Universidad de Buenos Aires, 1428 Buenos Aires, Argentina.
}

arms in galaxies [11], accretion disks [12], or the solar wind [13].

The paradigmatic instability in shear flows is the wellknown Kelvin-Helmholtz instability (KHI). It is an ideal hydrodynamic instability that converts the energy of the large-scale velocity gradients into kinetic and/or magnetic energy at much smaller scales, eventually driving a turbulent regime at these scales. The presence of a magnetic field parallel to the shear flow has a stabilizing effect and can even stall the instability if the shear velocity jump is smaller than twice the Alfven speed [14]. On the other hand, an external magnetic field perpendicular to the shear flow has no effect on the linear regime of the instability and it is simply advected by the flow. The Kelvin-Helmholtz instability plays an important role in several space physics and astrophysics problems, such as the interface between the solar wind and magnetospheres [15], coronal mass ejections [8], the stability of jet propagation [16], or cometary tails [17]. A general stability analysis in the presence of a magnetic field was carried out in Ref. [18] (see also Refs. [9] and [14]).

A relatively less known instability is the so-called Hall magnetoshear instability (Hall-MSI), which arises in plasmas embedded both in a shear flow and an external magnetic field perpendicular to the flow [19]. It is an ideal and microscopic instability, since it takes place at all wavelengths smaller than the ion skin depth. A linear study of Hall-MSI for weakly ionized plasmas has also been reported [20], which also includes the role of ambipolar diffusion. Hall-MSI arises only when the shear flow vorticity is antiparallel to the external magnetic field and corresponds to the destabilization of the ion-cyclotron wave mode. In other words, it arises whenever the shear is steep enough to be larger than the ion-cyclotron frequency [19] and therefore the free energy from the shear flow is invested in accelerating ions in their cyclotron motion. This instability might also play a role at the interface between astrophysical jets and their surrounding environment, just as it is also the case for KHI. Therefore, our goal in this paper is to set up a numerical experiment to allow these two instabilities (i.e., Hall-MSI and KHI) to compete. 
This paper is organized as follows. In Sec. II we present the so-called Hall-MHD equations, which are an extension of the traditional one-fluid MHD equations, which include the effect of the Hall current. In Sec. III we show these same equations in the case where the plasma is embedded both in an external large-scale shear flow and in a uniform magnetic field perpendicular to the flow. The shear flow is maintained by an external force that reaches an equilibrium with the viscous force. This exact equilibrium of the Hall-MHD equations is perturbed and its linear stability is studied in Sec. IV. Two competing instabilities are obtained: the macroscopic Kelvin-Helmholtz instability is studied in Sec. V, while the microscopic Hall-MSI instability is addressed in Sec. VI. A comparative study between the corresponding growth rates of these two instabilities is performed in Sec. VII. Finally, the conclusions of the present work are listed in Sec. VIII.

\section{HALL-MHD EQUATIONS}

The incompressible Hall-MHD equations for a fully ionized hydrogen plasma are the modified induction equation (i.e., with the addition of the Hall current) and the equation of motion (the Navier-Stokes equation),

$$
\begin{gathered}
\frac{\partial \boldsymbol{B}}{\partial t}=\nabla \times\left[\left(\boldsymbol{U}-\epsilon v_{A} \nabla \times \boldsymbol{B}\right) \times \boldsymbol{B}\right]+\eta \nabla^{2} \boldsymbol{B} \\
\frac{\partial \boldsymbol{U}}{\partial t}=-(\boldsymbol{U} \cdot \nabla) \boldsymbol{U}+v_{A}^{2}(\nabla \times \boldsymbol{B}) \times \boldsymbol{B}-\nabla P+v \nabla^{2} \boldsymbol{U}+\boldsymbol{F} .
\end{gathered}
$$

The velocity $\boldsymbol{U}$ is expressed in units of a characteristic speed $U_{0}$, the magnetic field $\boldsymbol{B}$ is in units of $B_{0}$, and we also assume a characteristic length scale $L_{0}$ and a spatially uniform particle density $n_{0}$. The assumption of incompressibility is valid provided that the plasma velocity associated with the instabilities being considered remains significantly smaller than both the Alfvén velocity and the speed of sound. Because of quasineutrality, the electron and the proton particle densities are equal, i.e., $n_{e}=n_{i}=n_{0}$. The (dimensionless) Alfven speed is then $v_{A}=B_{0} / \sqrt{4 \pi m_{i} n_{0}} U_{0}$, while $\eta$ and $v$ are respectively the dimensionless magnetic diffusivity and kinematic viscosity. The parameter $\epsilon$ is the dimensionless ion skin depth, and measures the relative strength of the Hall effect,

$$
\epsilon=\frac{c}{\omega_{p i} L_{0}}
$$

where $w_{p i}=\sqrt{4 \pi e^{2} n_{0} / m_{i}}$ is the ion plasma frequency.

These equations are complemented by the solenoidal conditions for both vector fields, i.e.,

$$
\nabla \cdot \boldsymbol{B}=0=\nabla \cdot \boldsymbol{U} .
$$

From a theoretical point of view, Hall-MHD corresponds to a two-fluid description of a fully ionized plasma: a positively charged ion species of mass $m_{i}$ moving with the velocity field $\boldsymbol{U}(\boldsymbol{r}, t)$, and negatively charged massless electrons with the velocity

$$
\boldsymbol{U}_{e}=\boldsymbol{U}-\epsilon v_{A} \nabla \times \boldsymbol{B},
$$

which stems from Ampère's Law (i.e., $\boldsymbol{J}=\frac{c}{4 \pi} \boldsymbol{\nabla} \times \boldsymbol{B}$ ) and from the expression for the electric current density for this two-fluid plasma: $\boldsymbol{J}=e n_{0}\left(\boldsymbol{U}-\boldsymbol{U}_{e}\right)$. Note that it is a simplified version of a two-fluid description, since we are neglecting the mass of electrons. For this reason, the smallest scales covered by this description have to remain much larger than the scale of electron inertia $c / \omega_{p e}\left(\omega_{p e}=\sqrt{4 \pi e^{2} n_{0} / m_{e}}\right.$ is the electron plasma frequency), which is determined by the electron mass $m_{e}$.

\section{SHEAR-DRIVEN HALL-MHD EQUATIONS}

Let us assume that the plasma is subjected to an externally applied shear flow given by

$$
\boldsymbol{U}_{0}=u_{0}(x) \hat{\boldsymbol{y}},
$$

so that the total velocity field is now $\boldsymbol{U}_{0}+\boldsymbol{U}$. Therefore, the Hall-MHD equations given in Eqs. (1)-(2) become

$$
\begin{gathered}
\frac{\partial \boldsymbol{B}}{\partial t}+u_{0}(x) \frac{\partial \boldsymbol{B}}{\partial y}-\frac{d u_{0}}{d x} B_{x} \hat{\boldsymbol{y}} \\
=\nabla \times\left[\left(\boldsymbol{U}-\epsilon v_{A} \nabla \times \boldsymbol{B}\right) \times \boldsymbol{B}\right]+\eta \nabla^{2} \boldsymbol{B} \\
\frac{\partial \boldsymbol{U}}{\partial t}+u_{0}(x) \frac{\partial \boldsymbol{U}}{\partial y}+\frac{d u_{0}}{d x} U_{x} \hat{\boldsymbol{y}} \\
=-(\boldsymbol{U} \cdot \nabla) \boldsymbol{U}+v_{A}^{2}(\nabla \times \boldsymbol{B}) \times \boldsymbol{B}-\nabla P+v \nabla^{2} \boldsymbol{U}+\boldsymbol{F} .
\end{gathered}
$$

Oftentimes such a shear flow is meant to simulate a large-scale velocity gradient acting on the relatively more microscopic degrees of freedom of the flow dynamics. We assume an imposed large-scale flow given by

$u_{0}(x)=U_{0}\left[\tanh \left(\frac{x-\frac{\pi}{2}}{\Delta}\right)-\tanh \left(\frac{x-\frac{3 \pi}{2}}{\Delta}\right)-1\right]$,

which corresponds to the encounter of largely uniform flows of intensities $+U_{0} \hat{\boldsymbol{y}}$ and $-U_{0} \hat{\boldsymbol{y}}$ through an interface of thickness $2 \Delta$ parallel to the flows. The configuration is sketched in Fig. 1, where the jump provided by the hyperbolic

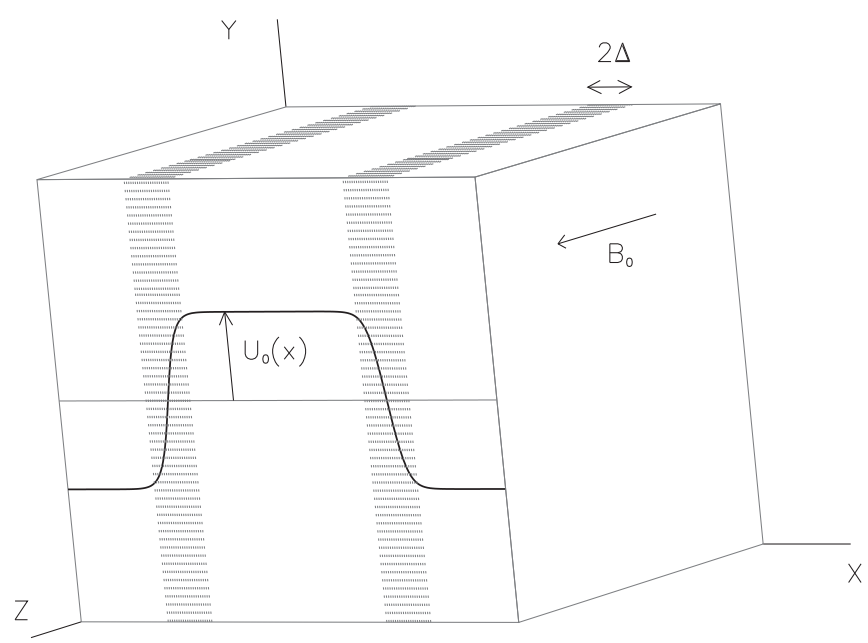

FIG. 1. Numerical box displaying the imposed velocity flow $U_{y}(x)$ and the external magnetic field $B_{0} \hat{z}$. The shaded patches correspond to regions with intense shear. Each axis ranges from $0-2 \pi$. 
tangent is duplicated to satisfy periodic boundary conditions throughout the numerical box.

The assumption of a hyperbolic tangent profile for shear flows with a finite thickness is standard practice in the literature $[9,21,22]$ as a way to study the evolution of such flows in a simplified configuration. The velocity profile given in Eq. (9) is an exact equilibrium of Eqs. (1)-(2) obtained through the application of the stationary external force $\boldsymbol{F}_{0}=$ $-v \nabla^{2} u_{0}(x) \hat{\boldsymbol{y}}$, in the absence of magnetic field (or more generally, in the presence of a uniform magnetic field along $\hat{z})$. Since the initial profile would slowly diffuse because of the effect of the viscous force, it will not be an exact equilibrium of the equation of motion. Our way out of this technical difficulty is therefore to apply a stationary force that reaches an equilibrium with the viscous force. In equilibrium, the work exerted by this force on the fluid exactly compensates for the viscous energy dissipation. In the ideal limit, this stationary force will become asymptotically zero. This strategy provides a reasonable numerical description of large-scale astrophysical flows for which the effect of viscosity is negligibly small. In practice, it amounts to situations such that the relevant time scales are much shorter than the diffusion time for the large-scale flow. We therefore apply this external force in our numerical box, to make sure that we are numerically studying the stability of the equilibrium given by the velocity profile of Eq. (9).

For sufficiently small parcels of fluid near the center of the shaded regions displayed in Fig. 1, the external shear can be approximated by a linear profile given by,

$$
\boldsymbol{U}_{0} \approx \frac{U_{0}}{\Delta}\left(x-x_{0}\right) \hat{\boldsymbol{y}}=\omega_{s h}\left(x-x_{0}\right) \hat{\boldsymbol{y}}
$$

which corresponds to a flow of constant vorticity of intensity $\omega_{s h}$ pointing in the $\hat{z}$ direction in the slice centered at $x_{0}=$ $\pi / 2$ (and constant vorticity $-\omega_{s h} \hat{z}$ in the slice centered at $x_{0}=3 \pi / 2$ ). The dynamics of plasmas embedded in linear shear profiles has been numerically studied using the so-called shearing-box simulations $[23,24]$. For the particular case of Hall-MHD flows, one-dimensional shearing-box simulations have also been reported to study shear-driven instabilities [19].

In what follows, we also assume the plasma to be immersed in a uniform magnetic field given by $B_{0} \hat{z}$, so that the total magnetic field is given by $B_{0} \hat{z}+\boldsymbol{B}$. As mentioned, the equilibrium velocity profile given by Eq. (9) is an exact solution of Eqs. (1)-(2) even in the presence of a uniform magnetic field $B_{0} \hat{z}$ and under the action of the external force $\boldsymbol{F}_{0}=-v \nabla^{2} u_{0}(x) \hat{\boldsymbol{y}}$. Therefore, the vector fields $\boldsymbol{U}$ and $\boldsymbol{B}$ hereafter correspond to the departures from this exact equilibrium.

\section{LINEARIZED HALL-MHD EQUATIONS}

The linearised version of Eqs. (7)-(8) to describe the dynamics of the perturbative components $\boldsymbol{U}$ and $\boldsymbol{B}$ are

$$
\begin{aligned}
\frac{\partial \boldsymbol{B}}{\partial t} & +u_{0} \frac{\partial \boldsymbol{B}}{\partial y}-u_{0}^{\prime} B_{x} \hat{\boldsymbol{y}} \\
& =\nabla \times\left[\left(\boldsymbol{U}-\epsilon v_{A} \nabla \times \boldsymbol{B}\right) \times \hat{z}\right]+\eta \nabla^{2} \boldsymbol{B}
\end{aligned}
$$

$$
\begin{aligned}
& \frac{\partial \boldsymbol{U}}{\partial t}+u_{0} \frac{\partial \boldsymbol{U}}{\partial y}+u_{0}^{\prime} U_{x} \hat{\boldsymbol{y}} \\
& =v_{A}^{2}(\nabla \times \boldsymbol{B}) \times \hat{\boldsymbol{z}}-\nabla P+v \nabla^{2} \boldsymbol{U}
\end{aligned}
$$

where $u_{0}^{\prime}$ expresses the spatial derivative of the profile $u_{0}(x)$.

This linear set of equations contains two competing instabilities: the hydrodynamic and large-scale Kelvin-Helmholtz instability, and the magnetohydrodynamic and small-scale Hall-MSI instability. Both of them are shear-driven instabilities, i.e., they arise in the shaded regions shown in Fig. 1. In the next two sections we summarize the basic features of each of these instabilities.

\section{KELVIN-HELMHOLTZ INSTABILITY (KHI)}

A shear flow such as the one given by Eq. (9) is subjected to the well-known Kelvin-Helmholtz instability (KHI), which is of a purely hydrodynamic nature, i.e., it occurs even in the absence of any magnetic field. Within the framework of MHD, the stability of a tangential velocity discontinuity (i.e. in the limit of $\Delta=0$ ) was first studied by Ref. [9]. For the case of an external magnetic field aligned with the shear flow, the mode is stabilized by the magnetic field, unless the velocity jump exceeds twice the Alfvén speed. For the case we are interested in, i.e., an external magnetic field perpendicular to the shear flow (see Fig. 1), the magnetic field has no effect and the flow is unstable for all velocity jump intensities.

A stability analysis of a sheared MHD flow of finite thickness (i.e., $\Delta \neq 0$ ) in a compressible plasma has also been performed [18], confirming the result that an external magnetic field perpendicular to the shear flow has no effect on the KHI, i.e., it reduces to the hydrodynamic case. If we approximate the hyperbolic tangent profile given in Eq. (9) by piecewise linear functions, the instability growth rate arising from Eq. (12) is (see Ref. [25])

$$
\gamma_{k h}^{2}=\frac{1}{4 \Delta^{2}}\left(e^{-4 k_{y} \Delta}-\left(2 k_{y} \Delta-1\right)^{2}\right),
$$

which attains its maximum at $\lambda_{\max } \approx 15.7 \Delta$ and $\gamma_{k h \text {, max }} \approx$ $0.2 / \Delta$. In our dimensionless units, the numerical box has linear size $2 \pi$. We set $\Delta=0.1$ so that the instability rate peaks at $\lambda_{\text {max }} \approx \pi / 2$.

We perform a numerical integration of Eqs. (7)-(8) subjected to the shear profile given in Eq. (9) on the cubic box of linear size $2 \pi$ sketched in Fig. 1, assuming periodic boundary conditions in all three directions. The number of grid points is $256^{3}$ and the dimensionless Alfven speed was set at $v_{A}=1$ in all our simulations, indicating that the external magnetic field intensity $B_{0}$ is such that its Alfven velocity is comparable to the maximum velocity $U_{0}$ of the shear profile. The values of the dimensionless parameters required for these simulations are listed in Table I, both for purely MHD simulations (i.e., $\epsilon=0$ ) and for HMHD simulations. We use a pseudospectral strategy to perform the spatial derivatives and a second-order Runge-Kutta scheme for the time integration (see a detailed description of the code in Ref. [26]). For the viscosity and resistivity coefficients we chose $v=\eta=2 \times 10^{-3}$ (see Table I), which are small enough to produce energy dissipation only at very small scales, comparable to the Nyquist wave number. In all simulations, the pressure in Eq. (2) is obtained 
TABLE I. Values of dimensionless parameters for runs MHD and HMHD: $v_{A}$ is the Alfvén speed, $\Delta$ is the initial thickness of the shear layer, $\eta$ is the magnetic diffusivity, $v$ is the kinematic viscosity, and $\epsilon$ is the the ion skin depth.

\begin{tabular}{lccccc}
\hline \hline Run & $v_{A}$ & $\Delta$ & $\eta$ & $v$ & $\epsilon$ \\
\hline MHD & 1 & 0.1 & $2.10^{-3}$ & $2.10^{-3}$ & 0.0 \\
HMHD & 1 & 0.1 & $2.10^{-3}$ & $2.10^{-3}$ & 0.4 \\
\hline \hline
\end{tabular}

self-consistently by taking the divergence of the equation, using the incompressibility condition, and solving at each time step the resulting Poisson equation for the pressure.

The evolution of the $\hat{z}$ component of vorticity is shown in Fig. 2 at two different times for an MHD run $(\epsilon=0$, see Table I). To estimate the instability growth rate, we use the component $U_{x}$ evaluated at $x_{0}=\pi / 2,3 \pi / 2$ (i.e., in the central part of the shear flows) as a proxy. In Fig. 3 we show the rms value of $\left\langle U_{x}^{2}\right\rangle_{y, z}\left(x_{0}, t\right)$ (i.e., averaging over all values on the $y, z$ plane) vs time,

$$
\begin{aligned}
U_{x, r m s}^{2}\left(x_{0}, t\right) & =\left\langle U_{x}^{2}\right\rangle_{y, z}\left(x_{0}, t\right) \\
& =\int_{0}^{2 \pi} d y \int_{0}^{2 \pi} d z U_{x}^{2}\left(x_{0}, y, z, t\right) .
\end{aligned}
$$

The thin black trace corresponds to $x_{0}=\pi / 2$, while the thick gray trace corresponds to $x_{0}=3 \pi / 2$, although (as expected) the two curves are almost undistinguishable.

Our best fit to this exponential growth, corresponds to $\gamma_{k h} \approx$ 1.8 , which is fully consistent with the theoretical value given in Eq. (13), considering that the spatial spectral content of $U_{x}\left(x_{0}=\pi / 2, y, z\right)$ in terms of $k_{y}$ is peaked at $k_{y}=2,3$.

\section{HALL MAGNETOSHEAR INSTABILITY (HALL-MSI)}

In its simplest version, the Hall-MSI instability arises on $x, z$ planes of the configuration depicted in Fig. 1, i.e., assuming translational symmetry along the $\hat{\boldsymbol{y}}$ direction $\left(\partial_{y}=0\right)$. For this instability to occur, we need the Hall term to be non-negligible $[\epsilon \neq 0$ in Eq. (11)] and also an intense shear. Therefore, this instability will be spatially localized around $x_{0}=\pi / 2$ and
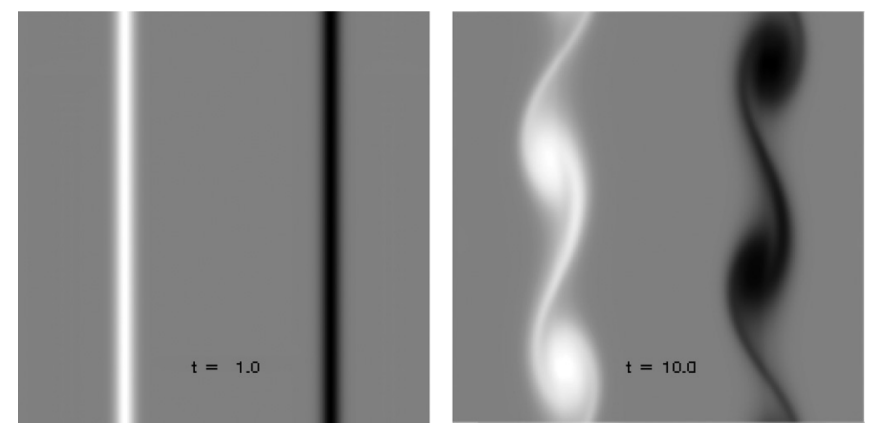

FIG. 2. Distribution of $\omega_{z}$ on the $x, y$ plane for the MHD run $(x$ is the horizontal axis and each axis ranges from 0 to $2 \pi$ ) at an early time $t=1$ (left frame) and also at a later time $t=10$ (right frame), where the Kelvin-Helmholtz instability has entered a nonlinear stage. Light (dark) regions correspond to structures of strong positive (negative) vorticity $\omega_{z}$.

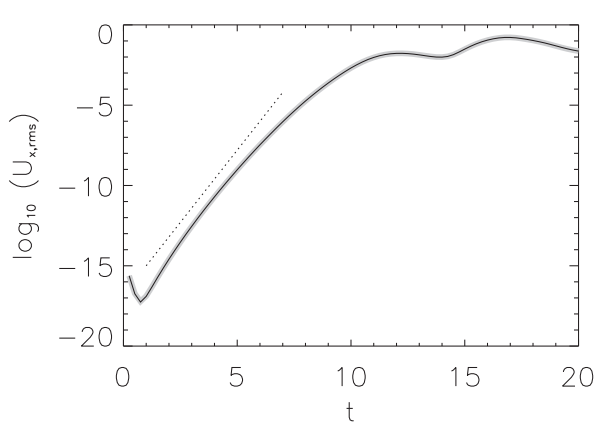

FIG. 3. Root mean square value of $\left\langle U_{x}^{2}\right\rangle_{y, z}\left(x_{0}, t\right)$ vs time in a lin-log plot for the MHD run (i.e., $\epsilon=0$ ). The thin black trace corresponds to $x_{0}=\pi / 2$ and the thick gray trace to $x_{0}=3 \pi / 2$. Superimposed we show a fit corresponding to $\gamma_{k h} \approx 1.8$.

$x_{0}=3 \pi / 2$ (i.e., the shaded slices shown in Fig. 1). Within these slices, we approximate the imposed shear flow by a linear profile characterized by a constant external vorticity $\omega_{s h} \hat{z}$, as shown in Eq. (10). Note that $\omega_{s h}=+U_{0} / \Delta$ at $x_{0}=$ $\pi / 2$, corresponding to a vorticity vector field aligned with the external magnetic field $B_{0} \hat{z}$. On the other hand $\omega_{s h}=-U_{0} / \Delta$ at $x_{0}=3 \pi / 2$, which implies that vorticity is antiparallel to the magnetic field in this slice. Under these considerations, the linear equations (11)-(12) lead to the following dispersion relation:

$$
\begin{aligned}
& \left(\frac{\gamma}{v_{A} k_{z}}\right)^{4}+\left(\frac{\gamma}{v_{A} k_{z}}\right)^{2}\left(2+\frac{\omega_{s h} \epsilon}{v_{A}}+\epsilon^{2} k^{2}\right)+\left(1+\frac{\omega_{s h} \epsilon}{v_{A}}\right) \\
& \quad=0
\end{aligned}
$$

where $k_{z} \neq 0$ and $k^{2}=k_{x}^{2}+k_{y}^{2}+k_{z}^{2}$ and $\omega=i \gamma$. We note that the dispersion relationship displayed in Eq. (15) has also been obtained [see Eq. (38) in Ref. [20] for weakly ionized plasmas embedded in shear flows in the limit of asymptotically large density of neutrals, for which ambipolar diffusion becomes negligible in comparison with the Hall term [20]. The solutions of Eq. (15) are

$$
\begin{aligned}
\left(\frac{\gamma}{v_{A} k_{z}}\right)^{2}= & -\left(1+\frac{\omega_{s h} \epsilon}{2 v_{A}}+\frac{1}{2} \epsilon^{2} k^{2}\right) \\
& \pm \sqrt{\left(1+\frac{\omega_{s h} \epsilon}{2 v_{A}}+\frac{1}{2} \epsilon^{2} k^{2}\right)^{2}-\left(1+\frac{\omega_{s h} \epsilon}{v_{A}}\right)}
\end{aligned}
$$

In the absence of shear (i.e., $\omega_{s h}=0$ ) this dispersion relation does not correspond to any instability, since its solutions satisfy $\gamma^{2}<0$. The solutions for $\omega_{s h}=0$ are shown by the two black lines in Fig. 4 and in fact the positive branch in Eq. (16) corresponds to the propagation of whistlers (right-hand circularly polarized, upper black line) while the negative branch corresponds to ion-cyclotron waves (left-hand polarized, lower black line), which are the normal modes for incompressible Hall-MHD [27]. These modes propagate in any arbitrary direction, except those exactly perpendicular to the external magnetic field (since $k_{z} \neq 0$ ). 


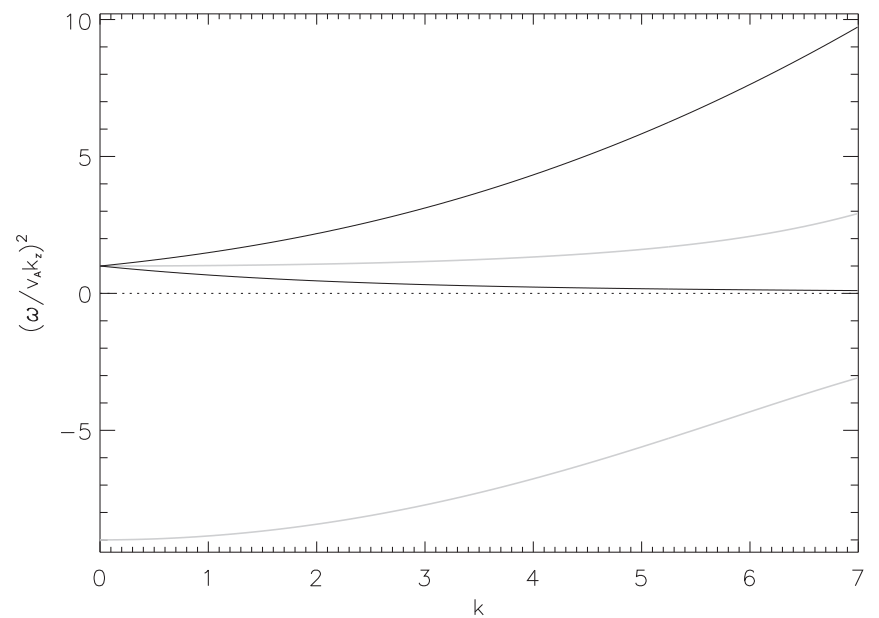

FIG. 4. Black lines show $\left(\omega / v_{A} k_{z}\right)^{2}$ versus $k$ for the normal modes in the absence of shear (i.e., $\omega_{s h}=0$ ). The upper branch corresponds to whistlers, while the lower one corresponds to the ion-cyclotron mode. The gray lines show the modified solutions in the presence of shear, more specifically for $\omega_{s h}=-10$. The negative branch corresponds to unstable evolutions, since $\omega^{2}<0$.

The necessary and sufficient condition for instability is that the last term in Eq. (15) becomes negative, namely that

$$
\omega_{s h}<-\frac{v_{A}}{\epsilon},
$$

which renders the ion-cyclotron branch unstable. The gray curves in Fig. 4 show the solutions of the dispersion relation [see Eq. (15)] modified by the presence of shear, more specifically for $\omega_{s h}=-10$. The Hall-MSI arises whenever any of the gray branches in Fig. 4 becomes negative, since that condition would correspond to $\omega^{2}<0$ (equivalent to $\omega= \pm i \gamma$ for $\gamma>0)$. This condition can only be satisfied on the slice centered at $x_{0}=3 \pi / 2$, since its vorticity is negative, and not on the slice located at $x_{0}=\pi / 2$ (since $\omega_{s h}>0$ within that slice). Also, only the ion-cyclotron branch leads to instability, while the whistler branch remains as a propagating mode, regardless of the intensity and orientation of the shear flow. Note that although this dispersion relation was correctly obtained by Kunz [20], our interpretation on the occurrence of the instability differs from the one provided in that paper. The argument exhibited by Kunz relies exclusively on the role played by the magnetic fluctuations in the linearized induction equation [see his Eq. (46) and below]. Such an approximation would hold in the so-called electron MHD regime, corresponding to negligible kinetic energy in comparison to magnetic energy, for which only the whistler mode propagates. However, we find that Hall-MSI arises as a result of the destabilization of the ion-cyclotron branch (for which the kinetic energy of the fluctuations is comparable or larger than the magnetic energy) while the whistler branch always remains stable.

In Fig. 5 we show the spatial distribution of the $\hat{z}$ component of the current density at two separate times, clearly showing the growth of the Hall-MSI on the slice containing negative vorticity centered at $x_{0}=3 \pi / 2$ for a HMHD run (i.e., $\epsilon \neq 0$, see Table I). Since $U_{0}=1$ and $\Delta=0.1$, then $\omega_{s h}=-10$ at $x_{0}=3 \pi / 2$, thus satisfying Eq. (17). This same simulation is
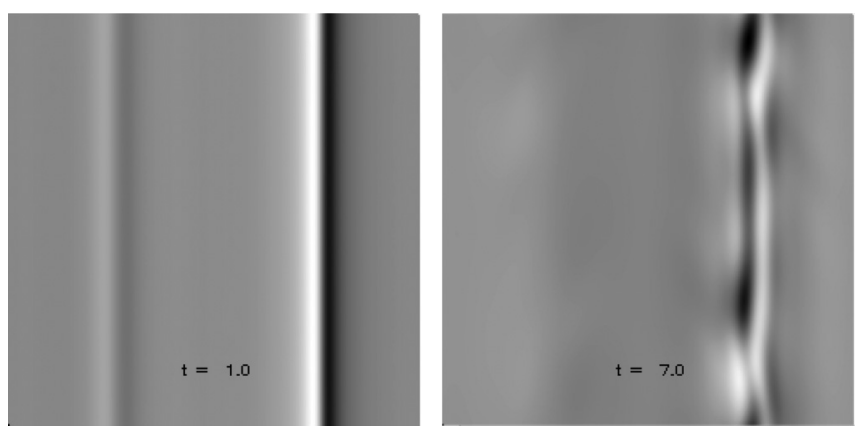

FIG. 5. Distribution of $J_{z}$ on the $x, y$ plane for the HMHD run ( $x$ is the horizontal axis and each axis ranges from 0 to $2 \pi$ ) at an early time $t=1$ (left frame) and also at a later time $t=7$ (right frame). Light (dark) regions correspond to structures of strong positive (negative) electric current density $J_{z}$.

also undergoing the Kelvin-Helmholtz instability, which can be observed in the distribution of $\omega_{z}(x, y)$ in Fig. 6. Note that even though Kelvin-Helmholtz evolves on both slices, as shown by the vorticity patterns in Fig. 6, the current density on the left slice (centered at $x_{0}=\pi / 2$ ) remains completely unaffected, as expected. Therefore, the current density pattern formed on the right slice (see the right panel of Fig. 6) is exclusively a consequence of the Hall-MSI.

According to the dispersion relation shown in Eq. (15) and once the instability condition given by Eq. (17) is satisfied, all wave numbers are unstable (see Fig. 4). The shear flow is localized, i.e., at $x_{0}=\pi / 2$ and $x_{0}=3 \pi / 2$, where

$$
\begin{aligned}
B_{r m s}^{2}\left(x_{0}, t\right) & =\left\langle|\boldsymbol{B}|^{2}\right\rangle_{y, z}\left(x_{0}, t\right) \\
& =\int_{0}^{2 \pi} d y \int_{0}^{2 \pi} d z|\boldsymbol{B}|^{2}\left(x_{0}, y, z, t\right) .
\end{aligned}
$$

Figure 7 shows $B_{r m s}\left(x_{0}=3 \pi / 2, t\right)$ as a function of time in a lin-log plot. We can observe the linear stage of the instability, where this function grows exponentially fast. The best fit corresponds to $\gamma_{h m s i} \approx 1.7$, which is also shown with a dotted trace. We also overlay $B_{r m s}\left(x_{0}=\pi / 2, t\right)$ using a gray trace, which does not reflect the Hall-MSI instability, since the slice centered at $x_{0}=\pi / 2$ does not satisfy the instability condition [i.e., the inequality shown in Eq. (17)].
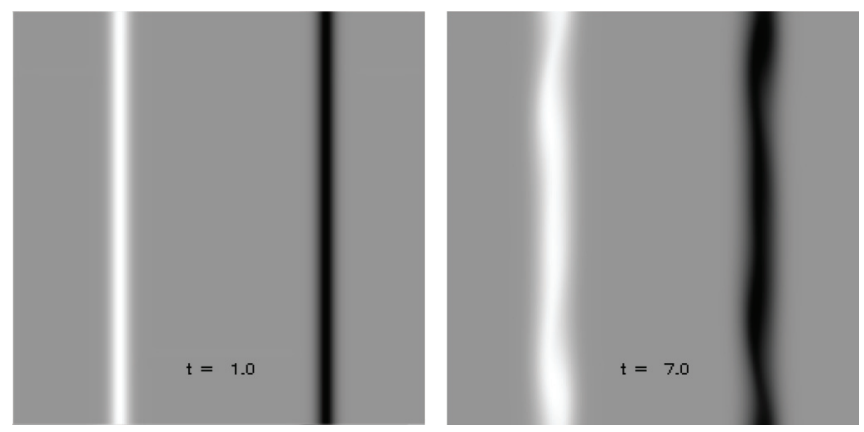

FIG. 6. Distribution of $\omega_{z}$ on the $(x, y)$ plane for the HMHD run ( $x$ is the horizontal axis and each axis ranges from 0 to $2 \pi$ ) at an early time $t=1$ (left frame) and also at a later time $t=7$ (right frame). Light (dark) regions correspond to structures of strong positive (negative) vorticity $\omega_{z}$. 


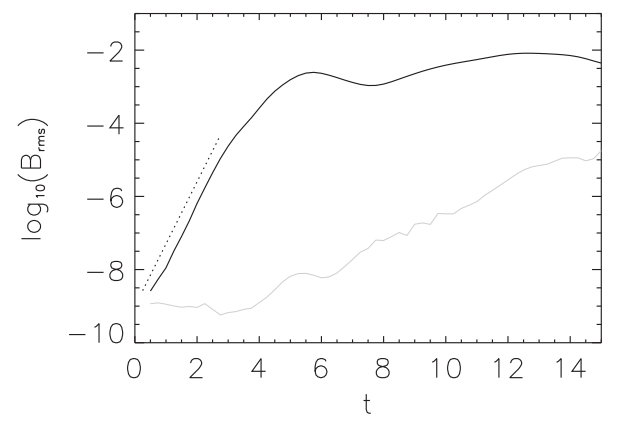

FIG. 7. Root mean square value of the magnetic field, i.e., the square root of $\left\langle|\boldsymbol{B}|^{2}\right\rangle_{y, z}\left(x_{0}=3 \pi / 2, t\right)$ vs. time in a lin-log plot for the HMHD run $(\epsilon=0.4)$. Superimposed we show a fit corresponding to $\gamma_{h m s i} \approx 1.7$. In gray trace we also show the square root of $\left\langle|\boldsymbol{B}|^{2}\right\rangle_{y, z}\left(x_{0}=\pi / 2, t\right)$.

Figure 7 also shows how the slice centered at $x_{0}=3 \pi / 2$ (black thin trace) gradually departs from the linear regime and the instability saturates giving rise to a stationary turbulent regime. The development of different regimes of Hall-MHD turbulence is studied elsewhere, and is beyond the scope of the present paper. For instance, Refs. [2,28] analyze the role of the Hall term on large-scale dynamos [29], perform a similar study on small-scale dynamos for different values of the magnetic Prandtl number, while Refs. [27,30] address the anisotropic nature of Hall-MHD turbulence in plasmas embedded in strong external magnetic fields. A detailed study of the role of all nonlinear terms of the Hall-MHD equations (including the Hall term itself) on the energy cascade arising on stationary turbulent regimes, is given in Ref. [1].

\section{DISCUSSION}

In Sec. V we showed that the Kelvin-Helmholtz instability arises for large-scale modes. From Eq. (13), we can readily obtain that the unstable modes satisfy $k \leqslant 0.64 / \Delta$ and also that $k=0.4 / \Delta$ is the most unstable mode, corresponding to

$$
\gamma_{k h, \max } \simeq 0.2 \omega_{s h} .
$$

On the other hand, the instability rate for Hall-MSI is obtained from the dispersion relation given in Eq. (15). When the instability condition given by Eq. (17) is satisfied, all wave numbers become unstable. The asymptotic value of the instability rate at large wave numbers is

$$
\gamma_{h m s i, \max } \simeq \frac{v_{A}}{\epsilon} \sqrt{\left|\omega_{s h}\right| \frac{\epsilon}{v_{A}}-1} .
$$

Since the ratio $v_{A} / \epsilon$ is equal to the dimensionless version of the ion-cyclotron frequency, i.e.,

$$
\frac{v_{A}}{\epsilon}=\omega_{c i} \frac{L_{0}}{U_{0}}, \quad \omega_{c i}=\frac{e B_{0}}{m_{i} c}
$$

the relative importance between both instability rates, depends solely on the ratio $R=\left|\omega_{s h}\right| / \omega_{c i}$. Figure 8 displays the ratio between the instability rates $\gamma_{h m s i, \text { max }}$ and $\gamma_{k h \text {,max }}$ as a function of $R$, showing that Hall-MSI grows faster than $\mathrm{KH}$ for all values of $R$ such that $1.04<R<23.96$. The instability condition for Hall-MSI given in Eq. (17) is equivalent to

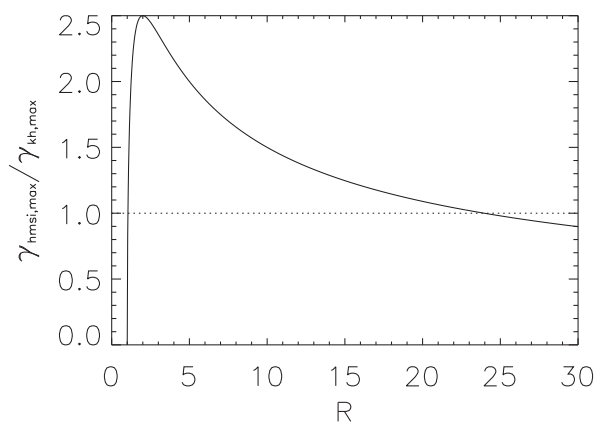

FIG. 8. Ratio of instability rates versus $R=\left|\omega_{s h}\right| / \omega_{c i}$.

$R>1$, and now we find that for $R>1.04$ it is already growing faster than KH. For $R=2$, the ratio of instability rates reaches a maximum, so that Hall-MSI is 2.5 times more unstable than KH. Hall-MSI remains more unstable up to $R=23.96$. However, note that at large values of $R$, we are moving in the direction of smaller spatial scales, where other kinetic effects not considered in this analysis might also become relevant.

The parameter $R$ was defined as a ratio of temporal frequencies, but at least for the case $v_{A}=1$ can also be regarded as a ratio of length scales. Since $\omega_{s h}=U_{0} / \Delta$ and $\epsilon=$ $c /\left(\omega_{p i} L_{0}\right)$, then $R=c /\left(\omega_{p i} \Delta v_{A}\right)$. For $v_{A}=1, R$ is simply the ratio between the ion skin depth $c / \omega_{p i}$ and the half thickness $\Delta$ of the slice. Therefore, for Hall-MSI to occur, we need the half thickness of the shear flow to be somewhat thinner than the ion skin depth. The thickness of shear flows will depend on the particular problem, and there are several mechanisms in astrophysics and space physics that may generate shear at very small scales and therefore might drive Hall-MSI. For instance, a whole range of sizes will spontaneously develop in turbulent flows down to the dissipative structures where viscosity becomes dominant. This is the case in the solar wind, where fluctuations are observed well below the ion skin depth. Besides turbulence, other examples of thin shear flows are differential rotation in accretion disks [31], zonal flows in drift wave turbulence in tokamaks [32], or magnetic reconnection in shear flows [33].

Note that the ratio of instability rates depicted in Fig. 8 corresponds to the idealized case where the KHI is dominated by its most unstable mode and Hall-MSI is led by very small wavelength modes. In general, both $\gamma_{k h}$ and $\gamma_{h m s i}$ will depend explicitly on the wave number, and therefore the ratio $R$ will be determined also by the initial condition. For instance, for the simulations shown in this work (corresponding to $R \approx 4$ ), both instability rates turned out to be comparable, while according to Fig. 8, the ratio would be different from unity.

\section{CONCLUSIONS}

In the present paper, we performed a comparative study of two competing shear-driven instabilities in a fully ionized plasma: Kelvin-Helmholtz and the Hall magnetoshear instability. Kelvin-Helmholtz is probably the paradigm of sheardriven instabilities, which leads to a large-scale corrugation of the shear layer, regardless of the presence of perpendicular magnetic fields. This instability has been invoked to play a role in several astrophysical plasmas, such as near the boundaries 
between astrophysical jets and the interstellar surroundings. On the other hand, in sufficiently low-density plasmas, also the Hall magnetoshear instability can take place, in which the Hall effect and the presence of a magnetic field play essential roles. Therefore, we carried out three-dimensional simulations of the Hall-MHD equations, starting from configurations such that these two instabilities are present.

The main result reported in this paper is that when the shear flow is intense enough that its central vorticity surpasses the ion-cyclotron frequency of the plasma, the Hall magnetoshear instability becomes non-negligible. Furthermore, we show that
Hall-MSI has growth rates larger than those for KHI for a wide range of values of the parameter $R$, which is the ratio between the vorticity at the center of the shear layer and the ion-cyclotron frequency of the plasma. We therefore believe that this result might have an impact on several astrophysical shear flows, such as the above mentioned example of astrophysical jets. This unexpected result is a direct consequence of the existence of a relatively new instability (namely, Hall-MSI, see Ref. [20], also Ref. [19]), which shows the potential relevance of the Hall effect in highly sheared plasma flows.
[1] P. Mininni, A. Alexakis, and A. Pouquet, J. Plasma Phys. 73, 377 (2007).

[2] P. Mininni, D. Gómez, and S. Mahajan, Astrophys. J. 619, 1019 (2005).

[3] P. Mininni, D. Gómez, and S. Mahajan, Astrophys. J. 567, L81 (2002).

[4] S. Donato et al., Phys. Plasmas 19, 092307 (2012).

[5] S. Donato, A. Greco, W. Matthaeus, S. Servidio, and P. Dmitruk, J. Geophys. Res. 118, 4033 (2013).

[6] G. Bodo, S. Massaglia, A. Ferrari, and E. Trussoni, Astron. \& Astrophys. 283, 655 (1994).

[7] A. Hasegawa, Adv. Phys. 34, 1 (1985).

[8] C. Foullon, E. Verwichte, K. Nykyri, M. Aschwanden, and I. Hannah, Astrophys. J. 767, 170 (2013).

[9] S. Chandrasekhar, Hydrodynamic and Hydromagnetic Stability (Oxford University Press, New York, 1961).

[10] M. Begelman, R. Blandford, and M. Rees, Rev. Mod. Phys. 56, 255 (1984).

[11] V. Dwarkadas and S. Balbus, Astrophys. J. 467, 87 (1996).

[12] S. Balbus and J. Hawley, Rev. Mod. Phys. 70, 1 (1998).

[13] S. Poedts, A. Rogava, and S. Mahajan, Astrophys. J. 505, 369 (1998).

[14] Y. Lau and C. Liu, Phys. Fluids 23, 939 (1980).

[15] E. N. Parker, Astrophys. J. 128, 664 (1958).

[16] M. Birkinshaw, in Advanced Topics on Astrophysical and Space Plasmas, edited by E. G. Dalpino, A. Peratt, G. M. Tanco, and A. Chian (Kluwer, Dordrecht, 1997), p. 17.
[17] J. C. Brandt and D. A. Mendis, in Solar System Plasma Physics, Vol. 2 (North-Holland Publ. Co., Amsterdam, 1979), p. 253.

[18] A. Miura and P. Pritchett, J. Geophys. Res. 87, 7431 (1982).

[19] C. Bejarano, D. Gómez, and A. Brandenburg, Astrophys. J. 737, 62 (2011).

[20] M. Kunz, Mon. Not. R.A.S. 385, 1494 (2008).

[21] P. Drazin, J. Fluid Mech. 4, 214 (1958).

[22] A. Miura, J. Geophys. Res. 97, 10655 (1992).

[23] J. Hawley, C. Gammie, and S. Balbus, Astrophys. J. 440, 742 (1995).

[24] A. Brandenburg and W. Dobler, Computer Phys. Comm. 147, 471 (2002).

[25] P. Drazin and W. Reid, Hydrodynamic Stability (Cambridge University Press, Cambridge, 1981).

[26] D. Gómez, P. Mininni, and P. Dmitruk, Phys. Scripta T116, 123 (2005).

[27] D. Gómez, S. Mahajan, and P. Dmitruk, Phys. Plasmas 15, 102303 (2008)

[28] P. Mininni, D. Gómez, and S. Mahajan, Astrophys. J. 587, 472 (2003).

[29] D. O. Gómez, P. D. Mininni, and P. Dmitruk, Phys. Rev. E 82, 036406 (2010).

[30] L. Martin, P. Dmitruk, and D. Gómez, Phys. Plasmas 17, 112304 (2010).

[31] S. Balbus and C. Terquem, Astrophys. J. 552, 235 (2001).

[32] A. Pushkarev, W. Bos, and S. Nazarenko, Phys. Plasmas 20, 042304 (2013).

[33] D. Knoll and J. Brackbill, Phys. Plasmas 9, 3775 (2002). 